\title{
Ultrasonography and Doppler Study to Predict Uterine Receptivity in Infertile Patients Undergoing Embryo Transfer
}

\author{
Mohd Shoeb Khan ${ }^{1} \cdot$ Aneesa Shaikh $^{1} \cdot$ Rekha Ratnani $^{2}$
}

Received: 15 April 2015/Accepted: 1 July 2015/Published online: 8 September 2015

(C) Federation of Obstetric \& Gynecological Societies of India 2015

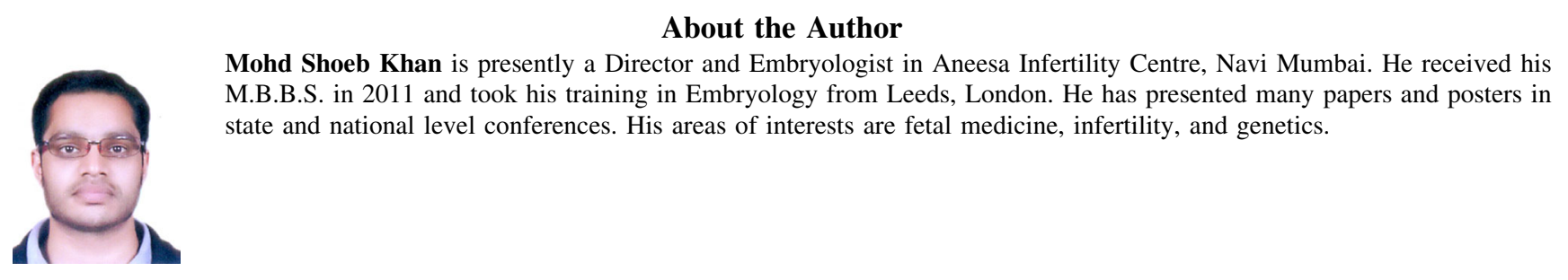

\begin{abstract}
Purpose To detect whether ultrasonographic parameters and Doppler analysis of uterine blood flow can be of value in the prediction of endometrial receptivity in infertile female patients undergoing embryo transfer.

Methods In this study, a total of 200 women with primary infertility undergoing embryo transfer were analyzed. Transvaginal ultrasonography was done on the 10th day of the menstrual cycle. To assess the uterine receptivity, we
\end{abstract}

Dr. Mohd Shoeb Khan is Director and Embryologist in Aneesa Infertility Centre, Navi Mumbai. Dr. Aneesa Shaikh is a sonologist in Aneesa Infertility Centre, Navi Mumbai. Dr. Rekha Ratnani is an infertility specialist in Apollo BSR Hospital, Bhilai.

Rekha Ratnani

rekharatnani@gmail.com

1 Aneesa Infertility Centre, Navi Mumbai, India

2 Apollo BSR Hospitals, Junwani Road, Bhilai 490020, India analyzed all the ultrasonographic and Doppler parameters. The slightly modified version of Applebaum Uterine Scoring System was used. This uterine scoring system included all the following parameters: endometrial thickness, endometrial morphology, endometrial blood flow within zone 3, myometrial echogenecity, uterine artery pulsatility index (PI), end diastolic blood flow, and myometrial blood flow internal to the arcuate vessels seen on gray-scale examination.

Results The pregnancy rates were higher in women with thick, distinct five-line endometrium and multifocal endometrial vascularity within zone 3 . Absent endometrial flow, despite highest values for the other parameters, was associated with no conception. In our study, no pregnancy occurred with uterine PI values of more than 2.8. Absence or reversal of end diastolic blood flow was associated with no conception. A maximum score of 20 was associated with the pregnancy rate of $97.4 \%$, whereas scores of 13 or less resulted in no pregnancies. 
Conclusion Uterine scoring system will help to perform embryo transfers in only favorable uteri and postpone or cancel those cycles in which poor uterine score is demonstrated.

Keywords Embryo transfer · Infertility . Uterine scoring system

\section{Introduction}

Infertility (clinical definition) is currently defined as 1 year of unwanted nonconception with unprotected intercourse in the fertile phase of the menstrual cycle [1]. Fertility treatment may be medical, surgical or involve assisted conception where by the egg and sperm are brought into close proximity to facilitate fertilization. Successful implantation during in vitro fertilization (IVF) and embryo transfer depends on many factors including embryo quality and uterine receptivity. Endometrial receptivity is defined as a temporary unique sequence of factors that make the endometrium receptive to embryonic implantation. It is the window of time when the uterine environment is conducive to blastocyst acceptance and subsequent implantation.

Transvaginal ultrasonography plays an essential role in modern fertility management. Regulation of uterine and ovarian blood flow is an important aspect of reproductive physiology. Pulsed and color Doppler technology have been used to show that blood flow directed to the uterus and ovary undergoes important modification in relation to cyclic hormonal changes and can be easily detected using color Doppler. A more logical approach would be to analyze all the ultrasonographic and Doppler parameters together in order to assess uterine receptivity. Based on numerous studies all over the world on color flow dynamics of reproductive organs, various scoring systems and criteria have been devised to predict implantation of the embryos transferred in assisted reproductive technology (ART). It is now only implantation, which eludes scientific basis. To overcome this, various centers are increasing the number of embryos transferred, which often leads to multiple gestations and painful embryo reductions, and spontaneous early pregnancy loss. It becomes an absolute necessity to evaluate the uterus and endometrium by color Doppler imaging, so we can perform embryo transfers in only favorable uteri. The fertilized embryos not used can be frozen for use in a favorable cycle based on the various scoring criteria like the uterine biophysical profile (UBP) and uterine scoring system for reproduction (USSR) [2].

Our study is an endeavor to examine the ability of an ultrasonography score to assess uterine receptivity.

\section{Materials and Methods}

The study was conducted in the Infertility Centre, Navi Mumbai. The study group included 200 female patients with primary infertility. The study was conducted between June 2011 and April 2014. The age of patients in our study ranged from 24 to 43 years. The duration of infertility ranged from 2 to 20 years. Informed and written consent was taken. Patients with secondary infertility and patients with associated male subfertility and infertility were excluded from the study.

Ninety-one women undergoing frozen embryo transfer (FET) after the first stimulated IVF treatment were recruited. They were pretreated with antagonist protocol, i.e., from day two of menses, they were given human menopausal gonadotropin (hMG) for ovarian stimulation and once the follicle was $14 \mathrm{~mm}$ (around 7th-9th day), we started them on injection Cetrorelix (Antagon). Human chorionic gonadotropin (hCG) was given intramuscularly when the leading follicle reached $18 \mathrm{~mm}$ in diameter, and there were at least three follicles of $\geq 16 \mathrm{~mm}$ in diameter. Oocyte retrieval was scheduled $36 \mathrm{~h}$ after the hCG injection. Patients were advised to have three embryos replaced into the uterine cavity $48 \mathrm{~h}$ after the retrieval.

A total of 109 recipients undergoing oocyte donation cycles (31 recipients) and embryo donation cycles (78 recipients) were also analyzed. Patients were started on oral contraceptive pills one month prior to embryo transfer. On the 21st day, they were given Goserelin injection, and then oral contraceptives were stopped 7 days after the Zoladex injection. Once they got their menses, a baseline ultrasound scan was done on day two of their menses to see if the ovaries were suppressed and to measure the endometrial thickness, after which the patients were started on Estradiol valerate $(2 \mathrm{mg} /$ day t.d.s.) until the embryo transfer day.

All ultrasound measurements were performed using MEDISON machine, SONOACERS with 4-9 MHZ transvaginal probe and MINDRAY, DC-7 with 4-10 MHZ transvaginal probe. Transvaginal ultrasonography was done on the 10th day of the menstrual cycle. The ultrasound examination was performed after the patient had emptied her bladder, and in lithotomy position. The maximum thickness of the endometrium on both sides of the midline was measured in a longitudinal plane. The endometrial pattern visualized was designated as distinct five-line appearance, hazy five-line appearance, or no layering. In principle, the central echogenic line represents the uterine cavity; the outer lines represent the basal layer of the endometrium or the interface between the endometrium and the myometrium. The relatively hypo-echogenic regions between the two outer lines and the central line may represent the functional layer of the endometrium. 
Endometrial vascularization was assessed on a longitudinal scan of the uterus. The color gate was placed over the thickest part of the endometrium. The presence or absence of color in zone 3 of the endometrium (hypoechoic inner layer) was determined. Using color Doppler in the 2D mode, flow velocity waveforms were obtained from the ascending main branch of the uterine artery on the right and left sides of the cervix in a longitudinal plane before they entered the uterus. The pulsatility index (PI) is measured from the flow velocity waveforms as the systolic peak velocity minus end diastolic velocity divided by the mean. As there was no difference in uterine PI between the left and the right sides, the averaged uterine PI was given. The presence, absence, or reversal of end diastolic blood flow was recorded. The myometrium was studied on a longitudinal scan of the uterus. Myometrium was considered to be appropriate if completely homogeneous. Myometrial blood flow internal to the arcuate vessels was then assessed. The modified version of Applebaum Uterine Scoring System is shown in Table 1.

A urinary b-hCG analysis was performed 14 days after ET and was repeated on the 16th day. If it was positive, ultrasound examination was performed 10-14 days later to confirm intrauterine pregnancy and to determine the number of gestational sacs present. Only clinical pregnancies defined by the presence of one or more gestational sacs or the histological confirmation of gestational product in miscarriages were considered.

\section{Statistical Analysis}

Results were expressed in percentages. The results of the study were expressed as sensitivity, specificity, positive predictive value (PPV), and negative predictive value (NPV).

\section{Results}

Out of 200 infertile women, there were 131 pregnancies with the pregnancy rate being $65.5 \%$. The endometrial thickness was in the range of $>7$ to $\leq 9 \mathrm{~mm}$ in $53 \%$ of females, and this group showed the pregnancy rate of $54.71 \%$. The associations of endometrial thickness, morphology, and vascularization with the pregnancy rate are shown in Charts 1, 2, and 3, respectively. In our study, 185 women $(92.5 \%)$ had relatively homogeneous myometrium with the pregnancy rate of $66.48 \%$, and 15 women ( $7.5 \%)$ had coarse/inhomogeneous myometrium with the pregnancy rate of $53.33 \%$. The maximum PI of the uterine artery in the pregnant group was 2.8. The averaged uterine artery PI is shown in Table 2. Normal waveform of the uterine artery is shown in Fig. 1.
Table 1 Modified version of Applebaum Uterine Scoring System

\begin{tabular}{|c|c|}
\hline Parameter & Score \\
\hline \multicolumn{2}{|l|}{ Endometrial thickness } \\
\hline$\leq 7 \mathrm{~mm}$ & $=0$ \\
\hline$>7$ to $\leq 9 \mathrm{~mm}$ & $=2$ \\
\hline$>9$ to $\leq 14 \mathrm{~mm}$ & $=3$ \\
\hline$>14 \mathrm{~mm}$ & $=1$ \\
\hline \multicolumn{2}{|l|}{ Endometrial morphology } \\
\hline No layering & $=0$ \\
\hline Hazy five-line appearance & $=1$ \\
\hline Distinct five-line appearance & $=3$ \\
\hline \multicolumn{2}{|l|}{ Endometrial vascularization within zone 3} \\
\hline Absent & $=0$ \\
\hline Present, but sparse & $=2$ \\
\hline Present multi focally & $=5$ \\
\hline \multicolumn{2}{|l|}{ Myometrial echogenecity } \\
\hline Coarse/inhomogeneous echogenecity & $=1$ \\
\hline Relatively homogeneous echogenecity & $=2$ \\
\hline \multicolumn{2}{|l|}{ Uterine artery Doppler flow evaluated by PI } \\
\hline$\geq 3.0$ & $=0$ \\
\hline $2.5-2.99$ & $=0$ \\
\hline $2.2-2.49$ & $=1$ \\
\hline$\leq 2.19$ & $=2$ \\
\hline \multicolumn{2}{|c|}{ End diastolic blood flow of the uterine artery } \\
\hline Present & $=3$ \\
\hline Absent/reversal & $=0$ \\
\hline \multicolumn{2}{|l|}{ Myometrial blood flow } \\
\hline Absent/weakly present (minimal) & $=0$ \\
\hline Strongly present & $=2$ \\
\hline Total & 20 \\
\hline
\end{tabular}

The end diastolic blood flow of the uterine artery was present in 180 women $(90 \%)$ and the pregnancy rate in this group was $72.77 \%$. The absence or the reversal of end diastolic blood flow was observed in 20 females $(10 \%)$, and this group was associated with no conception. The myometrial blood flow was present in 168 women $(84 \%)$. The pregnancy rate in this group was $65.47 \%$. The myometrial blood flow was minimal in 32 women (16\%). The pregnancy rate in this group was $65.62 \%$. No significant difference was noted between pregnant and nonpregnant women.

\section{Discussion}

In our study, the pregnancy rate decreased as the endometrial thickness decreased. Similar findings were reported by Noyes et al. [3]. Kovacs et al. [4] also reported that increased endometrial thickness was significantly associated with higher pregnancy rates. In our study, no 
Chart 1 Endometrial thickness-pregnancy rate

Chart 2 Endometrial morphology—pregnancy rate

Chart 3 Endometrial

morphology-pregnancy rate
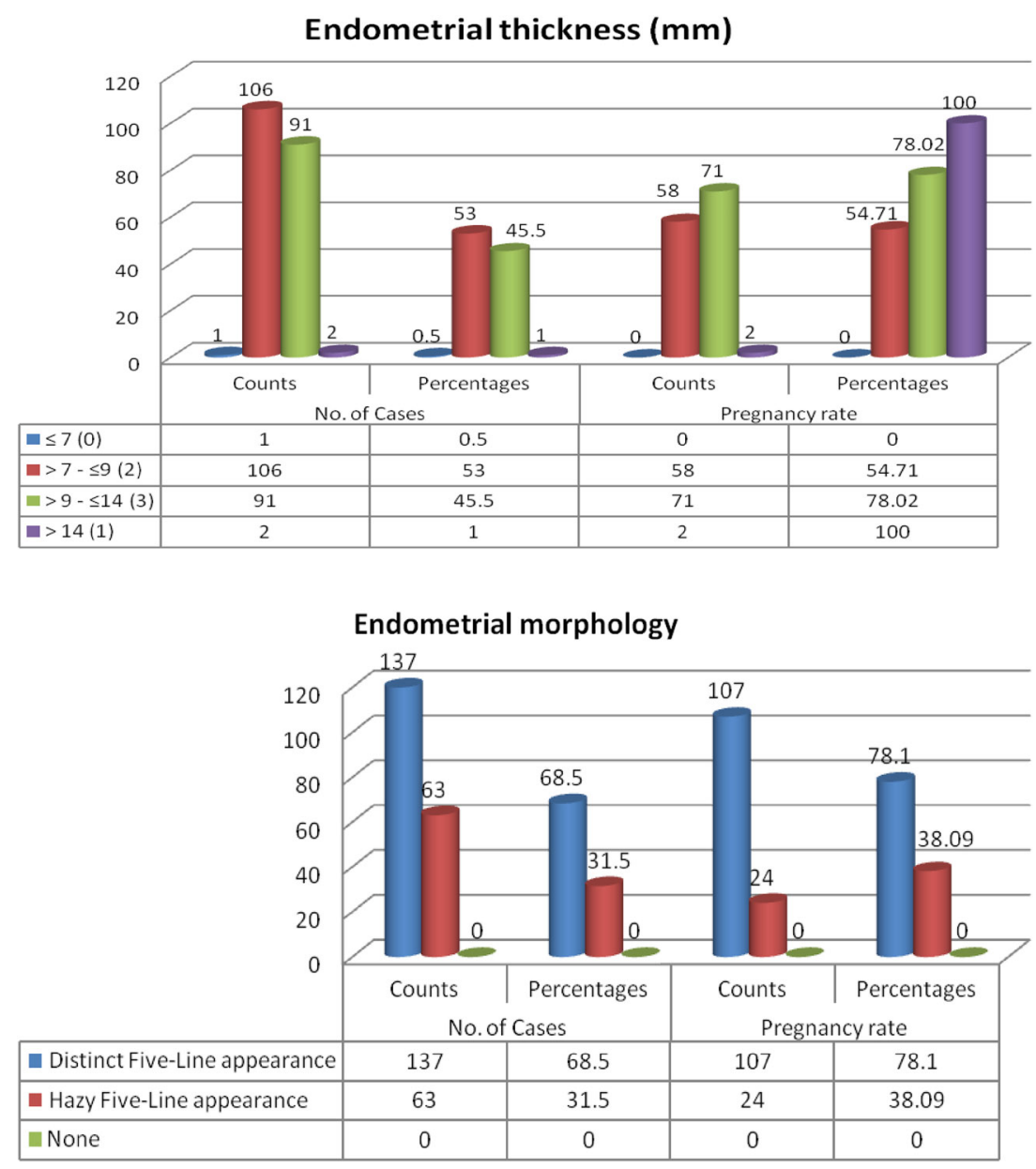

Table 2 The averaged uterine artery PI

\begin{tabular}{lllll}
\hline & Average & SD & Minimum & Maximum \\
\hline Pregnant & 1.72 & 0.43 & 0.7 & 2.8 \\
Non-pregnant & 2.27 & 0.58 & 1.0 & 3.7 \\
Total & 1.91 & 0.55 & 0.7 & 3.7 \\
\hline
\end{tabular}

pregnancy was reported when endometrial thickness was less than $7 \mathrm{~mm}$. Okohue et al. [5] concluded that endometrial thickness of at least $6.5 \mathrm{~mm}$ correlated with a higher rate of conception, while no pregnancy occurred in a similar study when the endometrial thickness was $<6 \mathrm{~mm}$. Casper RF speculated that thinner endometrium resulting in implantation failure may be related to oxygen tension. 
Fig. 1 Normal waveform of the uterine artery with low pulsatility index (1.49)

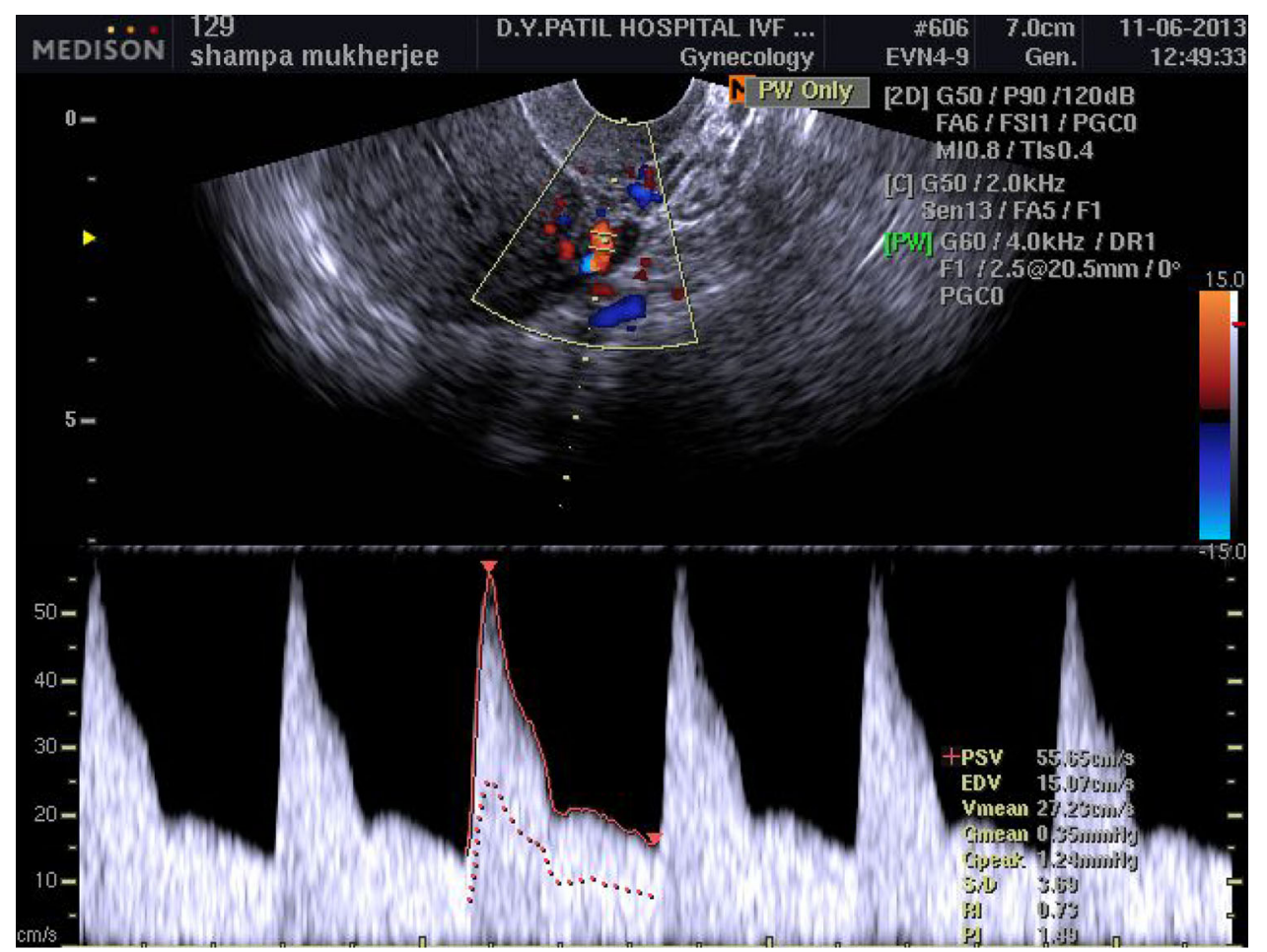

When the thickness measured by ultrasound is $<7 \mathrm{~mm}$, the functional layer is thin or absent, and the implanting embryo would be much closer to the spiral arteries and the higher vascularity and oxygen concentrations of the basal endometrium. The high oxygen concentrations near the basal layer could be detrimental compared with the usual low oxygen tension of the surface endometrium [6]. In a more recent study, clinical pregnancy was found to be positively associated with increased endometrial thickness and negatively associated with advanced age [7].

In our study, women with the distinct five-line appearance of the endometrium showed a higher pregnancy rate compared to that of hazy five-line appearance. Similar finding was reported by Merc et al. [8]. Zhao et al. concluded that endometrial thickness and pattern independently affect pregnant outcomes. Combined endometrial thickness and pattern could not predict the outcome of IVFET when endometrial thickness was $<7$ or $>14 \mathrm{~mm}$, while a triple-line pattern with a moderate endometrial thickness appeared to be associated with a good clinical outcome [9].

Successful implantation depends on a close interaction between the blastocyst and the receptive endometrium. A good blood supply toward the peri-implantation endometrium is usually considered to be an essential requirement for normal implantation [10]. Now, with the advance of ultrasonography, color Doppler imaging has been used in endometrial blood flow assessment. Color Doppler imaging enjoys the advantages of high sensitivity to slow blood flow, while being less dependent on angles and providing a lesscluttered image [11]. The endometrial and periendometrial areas are divided into the following four zones [12].

\section{Endometrial Blood Flow}

The endometrial and periendometrial areas are divided into the following four zones:

Zone 1: A 2-mm thick area surrounding the hyperechoic outer layer of the endometrium.

Zone 2: The hyperechoic outer layer of the endometrium. Zone 3: The hypoechoic inner layer of the endometrium. Zone 4: The endometrial cavity.

In our series, the pregnancy rate was significantly higher in patients with multifocal vascularity in zone 3. Similar finding was reported by Chien et al. [13]. A study done by Apollo-Assisted Reproduction Unit revealed the significant correlation between pregnancy rate and endometrial vascularity [14]. Absent endometrial vascularization was

Table 3 Sensitivity and specificity of scoring method

\begin{tabular}{llllllll}
\hline True positive & True negative & False positive & False negative & Sensitivity & Specificity & PPV & NPV \\
\hline 119 & 54 & 15 & 12 & 0.9083 & 0.7826 & 0.8880 & 0.8181 \\
\hline
\end{tabular}


associated with no conception. Our results are in agreement with Applebaum [2]. In the studies conducted by Chien et al. [13] and Maugey-Laulon et al. [15], absent endometrial vascularization was associated with significantly lower pregnancy rate. It has been postulated that a better endometrial and subendometrial vascularity can lead to a better placental development during pregnancy which is associated with a lower risk of miscarriage and a higher chance of live birth following ART [10]. The average PI of the uterine artery was higher was higher in nonconception than in conception cycles. In our series, no conception was reported when PI was more than 2.8. Tekay et al. reported no pregnancy when the PI was more than 3 [16].

Diastolic blood flow may be categorized as full or continuous and discontinuous, i.e., reduced or absent flow velocity. Good uterine perfusion, as shown by full diastolic blood flow with low resistance during the early and midsecretory phases, correlates with conception following assisted reproduction treatment. In our series, the absence or the reversal of end diastolic blood flow was associated with no conception. In a recent study, endometrium pattern, endometrium thickness, and end diastolic blood flow in combination was shown to be the most effective combination for evaluation of uterine receptivity [17].

In our study, a perfect score of 20 was associated with a pregnancy rate of $97.4 \%$. Scores of 17-19 were associated with a pregnancy rate of $85.4 \%$. Scores of 14-16 were associated with a pregnancy rate of $28.6 \%$. Applebaum [2] reported a pregnancy rate of $100 \%$ in females with a score of $20,80 \%$ in females with scores of $17-19$, and $60 \%$ in women with scores of 14-16. In our series, scores of 13 or less resulted in no pregnancies. Similar finding was reported by Applebaum [2]. The sensitivity and specificity of scoring method are shown in Table 3.

Sensitivity of 0.9083 and specificity of 0.7826 show that the scoring method can be used for the prediction of uterine receptivity in women undergoing embryo transfer.

\section{Conclusion}

The endometrial receptivity (thickness and vascularity) is a predeterminant in predicting the outcome of pregnancy in women. Uterine scoring system will help to perform embryo transfers in only favorable uteri and postpone or cancel those cycles in which poor uterine score is demonstrated.

\section{Compliance with Ethical Standards}

Conflict of interest The authors declare that there is no conflict of interest.

Ethical approval All procedures followed were in accordance with the ethical standards of the responsible committee on human experimentation (institutional and national) and with the Helsinki Declaration of 1975, as revised in 2008 (5).

Informed consent Informed consent was obtained from all patients for being included in the study.

\section{References}

1. Gnoth C, Godehardt E, Frank-Herrmann P, et al. Definition and prevalence of subfertility and infertility. Hum Reprod. 2005; 20(5):1144-7. doi:10.1093/humrep/deh870.

2. Applebaum M. The uterine biophysical profile. Ultrasound Obstet Gynecol. 1995;5(1):67-8.

3. Noyes N, Hampton BS, Berkeley A, et al. Factors useful in predicting the success of oocyte donation: a 3-year retrospective analysis. Fertil Steril. 2001;76(1):92-7.

4. Kovacs P, Matyas S, Boda K, et al. The effect of endometrial thickness on IVF/ICSI outcome. Hum Reprod. 2003;18(11):2337-41.

5. Okohue JE, Onuh SO, Ebeigbe P, et al. The effect of endometrial thickness on in vitro fertilization (IVF)-embryo transfer/intracytoplasmic sperm injection (ICSI) outcome. Afr J Reprod Health. 2009;13(1):113-21.

6. Casper RF. It's time to pay attention to the endometrium. Fertil Steril. 2011;96(3):519-21. doi:10.1016/j.fertnstert.2011.07.1096.

7. Zhang $\mathrm{X}$, Chen $\mathrm{CH}$, Confino $\mathrm{E}$, et al. Increased endometrial thickness is associated with improved treatment outcome for selected patients undergoing in vitro fertilization-embryo transfer. Fertil Steril. 2005;83(2):336-40.

8. Merc LT, Barco MJ, Bau S. 2D and 3D power Doppler ultrasound of endometrium as implantation marker. Donald Sch J Ultrasound Obstet Gynecol. 2008;2(2):1-11. doi:10.5005/jp-journals-10009-1052.

9. Zhao J, Zhang Q, Li Y. The effect of endometrial thickness and pattern measured by ultrasonography on pregnancy outcomes during IVF-ET cycles. Reprod Biol Endocrinol. 2012;28(10):100. doi:10.1186/1477-7827-10-100.

10. Ng EH, Chan CC, Tang OS, et al. Endometrial and subendometrial vascularity is higher in pregnant patients with livebirth following ART than in those who suffer a miscarriage. Hum Reprod. 2007;22(4):1134-41.

11. Wang L, Qiao J, Li R, et al. Role of endometrial blood flow assessment with color Doppler energy in predicting pregnancy outcome of IVF-ET cycles. Reprod Biol Endocrinol. 2010;18(8): 122. doi:10.1186/1477-7827-8-122.

12. Malhotra N, Malhotra J, Malhotra N, et al. Endometrial receptivity and scoring for prediction of implantation and newer markers. Donald Sch J Ultrasound Obstet Gynecol. 2010;4(4):439-46.

13. Chien LW, Au HK, Chen PL, et al. Assessment of uterine receptivity by the endometrial-subendometrial blood flow distribution pattern in women undergoing in vitro fertilization embryo transfer. Fertil Steril. 2002;78(2):245-51.

14. Bhadauriaa K, Sahnib R, Vermab S, et al. Colour Doppler ultrasound in controlled ovarian stimulation with intrauterine insemination. Apollo Med J. 2012;9(3):252-62.

15. Maugey-Laulon B, Commenges-Ducos M, Jullien V, et al. Endometrial vascularity and ongoing pregnancy after IVF. Eur J Obstet Gynecol Reprod Biol. 2002;104(2):137-43.

16. Tekay A, Martikainen H, Jouppilas P. The clinical value of transvaginal colour Doppler ultrasound in assisted reproductive technology procedures. Hum Reprod. 1996;11(8):1589-91.

17. Dechaund H, Bessueille E, Bousquet PJ, et al. Optimal timing of ultrasonographic and Doppler evaluation of uterine receptivity to implantation. Reprod Biomed Online. 2008;16(3):368-75. 